\title{
THE METHOD OF VALIDATION BY ZENITH
}

\author{
DAVID A. LEAVENS ${ }^{* 1}$ \\ *Corresponding Author: davidl@ sussex.ac.uk \\ ${ }^{1}$ School of Psychology, University of Sussex, Brighton, United Kingdom
}

A central question in consideration of the origins of language is to what extent linguistic communication reflects motivational and cognitive factors that evolved uniquely in the human lineage. Theorists have argued that humans have unique motivations for sharing attention and for collaborative action (shared intentionality), and uniquely human perceptions of psychological common ground, which are evident in the social interactions of human children, even before they master speech (e.g., Tomasello, Carpenter, \& Liszkowski, 2007). Others have claimed that humans have unique representational capacities that are manifested early in childhood (e.g., Povinelli, Bierschwale, and Čech, 1999). For over 20 years, these claims for human cognitive exceptionalism have relied on differences in response profiles between young humans and substantially older great apes when challenged with tests of their social awareness-age differences are confounded with species classifications (Leavens, Bard, \& Hopkins, 2017).

In this talk, I will describe the method of Validation by Zenith; this technique identifies a maximum capability response profile in humans, against which the performances of younger humans and animals can be compared. This method assumes that human adults respond to cognitive challenge with the most sophisticated psychological processes in the animal kingdom. This corrects for a bias that exists in the contemporary literature: performance differences between human children and older apes are interpreted as evidence of cognitive superiority of human children, but these interpretations are not validated against response profiles of human adults. If human adults respond similarly to human children in these tasks, then this validates interpretations of human children's cognitive superiority. On the other hand, if human adults behave similarly to apes, in a range of contemporary cognitive assays, then this refutes assumptions of the cognitive superiority of human children over older apes. 
For example, Povinelli et al. (1999) claimed that human children, but not chimpanzees, understood gaze direction as a referential signal. The human children were aged 3 years, whereas the chimpanzees were aged 6 years-twice as old as the human children. The general approach of this study was to challenge participants to use human adult behavioral cues to identify the location of hidden objects from two possible hiding locations. In a critical test condition, the experimenter turned to fixate a point significantly above the baited container, but in the same hemispace. In this condition the chimpanzees outperformed the human children, who performed at chance levels. The authors claimed two things about these results: first, they claimed that they had predicted the result in advance of the study on theoretical grounds and, second, they claimed that the higher performances of the chimpanzees reflected a "low-level" understanding of visual attention in others.

This interpretation was challenged by the later application of the method of Validation by Zenith, developed (albeit not named as such) by Thomas, Murphy, Pitt, Rivers, \& Leavens (2008). In Thomas et al., the experimenters made the assumption that human adults would exercise the maximum possible cognitive sophistication in the use of experimenter-given cues. They found that human adults, like the 6-year-old chimpanzees in Povinelli et al. (1999), successfully used the averted gaze to the correct hemisphere to locate hidden food.

I will describe more recent applications of the method of Validation by Zenith, which further support the conclusion that previous reports of cognitive differences between young humans and older apes implicate age differences, not evolutionarily based differences in cognitive skills in these kinds of cueing tasks.

\section{References}

Leavens, D. A., Bard, K. A., \& Hopkins, W. D. (2017). The mismeasure of ape social cognition. Animal Cognition. https://doi.org/10.1007/s10071-0171119-1 (Published online in advance of print.)

Povinelli, D. J., Bierschwale, D. T., \& Čech, C. G. (1999). Comprehension of seeing as a referential act in young children, but not juvenile chimpanzees. British Journal of Developmental Psychology, 17, 37-60.

Thomas, E., Murphy, M., Pitt, R., Rivers, A., \& Leavens, D. A. (2008). Understanding of visual attention by adult humans (Homo sapiens): A partial replication of Povinelli, Bierschwale, and Čech (1999). Journal of Comparative Psychology, 122, 428-436.

Tomasello, M, Carpenter, M., Liszkowski, U. (2007). A new look at infant pointing. Child Development, 78, 705-722. 